\title{
On the development of the isolation effect in free recall: Isolation by color
}

\author{
JOHN J. WINTERS and DAVID L. HOATS \\ E. R. Johnstone Training and Research Center, Bordentown, New Jersey
}

\begin{abstract}
In Experiment 1, three groups of subjects (ages 8, 11, and 14 years) read nine lists of nine words and immediately recalled each list. The middle item in each list was presented either in red (isolated) or in the same color as the other items (nonisolated). As age increased, the difference in recall between items isolated or nonisolated decreased, suggesting that the isolation effect and age are inversely related in free recall. Experiment 2 was conducted to determine whether this relationship was purely a function of responsiveness to the perceptual aspects of the stimuli, or was influenced also by memory capacity. Two more groups (ages 11 and 13) read lists 11 or 13 words long, respectively, with the middle item either isolated or not. The isolation effect was invariant over age. The proportion of yoked nonisolated items that were recalled varied with task difficulty, whereas the proportion of isolated items did not, thereby influencing the extent of the isolation effect. We concluded that, in this context, maturational changes in memory capacity influence the isolation effect.
\end{abstract}

The von Restorff phenomenon, also referred to as the isolation effect, is demonstrated when an item that is in some way unique or different from other items in a group is recalled significantly better than when it is the same as the other group items. That is, recall of the same item(s) is compared under two different experimental conditions. The proposed explanations for this phenomenon are described by Wallace (1965).

One of the explanations is that each item sets up its own neural trace. The homogeneous list items form an aggregate (background) of traces against which the heterogeneous item (figure) forms its own unique trace, resulting in superior recall compared with recall when the item is homogeneous. Another explanation maintains that generalization occurs between list items, causing interitem interference. Increasing the differentiation of an item will reduce interference of that item and facilitate its recall. Other explanations are offered in terms of mediation, of using organizational aids to enhance recall of the isolated item, of the surprise and attention-getting value of the isolated item, and, most recently, of the greater rehearsal of the isolated item. At the theoretical level, the causes of the isolation effect remain unresolved, and it is not clear which hypothesis is the most appropriate one under which set of circumstances. What is clear is that the effect is robust, is demonstrated when different isolating variables are used, and has been found in different population samples.

The authors are grateful to Sr. Helen Miller of St. Mary Elementary school in Bordentown, NJ, to Sr. Mary Louise Wynne of St. Raphael Elementary school in Trenton, NJ, to the respective faculties and parents, and to Heather Vail, for their invaluable assistance in obtaining subjects. We are also indebted to Herman H. Spitz for his critical reading of the manuscript, and to Maria Semchuk and Suzanne Borys for helping to untangle word clusters in the original lists. Requests for reprints should be sent to John J. Winters, E. R. Johnstone Training and Research Center, Bordentown, NJ 08505.
For almost a century, little attention has been given to whether the isolation effect changes during the course of development. Changes in the organization and rehearsal of information do occur as age increases (Flavell, 1970; Pezdek, 1983), but we have limited knowledge as to whether trace aggregation, differentiation, organization, surprise, rehearsal of items, or whatever, influences the degree of the isolation effect during development. However, determining which of the above affect this phenomenon is not our interest here. Rather, we are interested in examining the ways in which this phenomenon can increase our understanding of changes in memorial processing during development. The phenomenon is also of interest because of its applied aspects. For example, it occurs in our daily lives when unique, novel, or heterogeneous experiences are retrieved more readily from semantic memory than ordinary experiences are, and when unique experiences are stored more readily in episodic memory.

Color, which has been used as an isolating variable for 90 years (Calkins, 1894), has elicited superior recall of color-highlighted information throughout a large age range: 4-year-olds (Jones, 1965), 11-year-olds (Rabinowitz \& Andrews, 1973), 8th-graders (Hershberger \& Terry, 1965), high school students (Gabassi, 1980), and adults (Cashen \& Leicht, 1970). Consequently, color will be used here as the isolating variable.

Of the few studies in which isolation by color has been used with children and/or adolescents, only two have included more than one age group. Otto (1968) had 2nd-, 4th-, and 6th-graders learn English verbal responses to three-letter words written in Greek characters that were printed either in black or each in a different color. There were six pairs of items; learning occurred by means of the method of anticipation for both serial and scrambled presentation. There was some tendency for the youngest 
group to benefit most from the presentation of the colored as opposed to the black letters. This finding suggests that the isolation effect attenuates over the 4-year span investigated in that study.

In the Cimbalo, Nowak, and Soderstrom (1981) study (Experiment 2), 5-6- and 9-10-year-olds viewed seven outline pictures presented simultaneously on cards, with the middle picture colored pink when it was isolated. A probe technique required the child to indicate the location of the target picture when the cards were overturned. The younger group showed better recall of midposition items than did the older group when isolated and nonisolated items were combined. Recall of isolated items was significantly greater than recall of nonisolated items for the younger group, but not for the older group.

It is possible that the developmental trends found in these two studies were a function of the higher (asymptotic) recall performance by the older groups on the tasks, whether items were isolated or not, thereby reducing the relative difference in performance on isolated and nonisolated items. Since the tasks were identical for all age groups in each study, the level of difficulty most likely varied for the groups. Thus, developmental trends may be as much a function of storage capacity as it is of perception. Experiment 1 was conducted to examine the extent of the isolation effect in several age groups when the same task was given.

\section{EXPERIMENT 1}

\section{Method}

Subjects. There were 108 subjects, 36 in each of three age groups. The youngest group had a mean chronological age $(\overline{C A})$ of 8.80 years $(S D=.83)$, the next older group had a $\overline{C A}$ of 11.62 years $(.66)$, and the oldest group had a $\overline{C A}$ of 14.17 years (.47). The students in each age range were from Grades 2 through 4, 5 through 7, and 8 through 9 , respectively. Half of each group was in the control (experimental) condition and half of each of those groups was male (female). A younger group of 21 first-graders, who had a $\overline{C A}$ of 7.17 years (.35), was also tested. Of that group, only 9 could read all the words. The readers had a $\overline{C A}$ of 7.08 years (.39). This group's performance will be discussed separately.

Stimuli and Procedure. Two hundred monosyllabic nouns with $U$ values $>100$ were selected from the Carroll, Davies, and Richman (1971) collection. We selected 81 words that did not exceed the secondgrade level of difficulty, as judged by a reading specialist. This judgment was confirmed by the fact that all students at and above the secondgrade level could read all the words, with the exception of one secondgrader who was excluded from the study. The nouns were placed in nine lists with nine words per list in nine list orders. Each order differed from all other orders, in that each list in each order was in a different position in the list sequence and each word was in a different location in each list. We tried to avoid meaningful clusters of words (e.g., schoolhouse or moon-light) when assembling the lists.

The stimulus words were generated on a microcomputer terminal. A blue-green gel (Edmund Scientific Stock No. 60403, No. 877) was placed over each list as it appeared on the cathode-ray tube so that all items in the control list were the same blue color. Color slides were made of each nine-item list for a total of 81 slides. For the experimental condition, another set of 81 color slides of the lists was made, except that the middle word in each list was covered by an orange/amber gel (Edmund Scientific Stock No. 60403, No. 819) so that the isolated item was reproduced as red and the remaining items as blue.

The subjects were tested individually. They were seated with their eyes approximately $45 \mathrm{~cm}$ from the screen onto which each list was back- projected. The subjects were told that words would appear on the screen, that they were to read each word aloud, that the words would then disappear, and that they should recall as many list words as possible. As each list was exposed, the subjects read the nine words in each list sequentially from left to right at their own rate. After the ninth word was read, the experimenter immediately advanced the projector so that the screen was blank. A delay timer was simultaneously activated for $15 \mathrm{sec}$, during which time the subject recited all the words that the subject could recall from the list of words. It was found in a pilot study, using the same number of words, that $\mathbf{1 5} \mathrm{sec}$ was ample time for recall.

After the recall period, the timer activated the projector, which advanced the magazine to expose the next slide. This sequence of reading and recalling words occurred for nine lists for each subject. The list orders and design were identical in the experimental and control groups, apart from the exception that, for the experimental group, the middle word in each list was in the color red rather than blue.

\section{Results}

The percentage of items correctly recalled by each person at each position over the nine trials was determined and converted to its arcsin to normalize the distribution. The statistical analyses were performed on the transformed data.

A 3 (groups) $\times 2$ (conditions) $\times 9$ (serial positions) analysis of variance, with repeated measures on the last variable, revealed that the groups differed in the percentage of items correctly recalled $[F(2,102)=17.47]$. (All reported statistics are significant at least at the .05 level.) Serial positions also differed $[F(8,816)=35.69]$, as did the interaction between conditions and serial position $[F(8,816)=$ 2.98]. Multiple comparison tests (Tukey HSD) indicated that the two older groups recalled a higher percentage of items than did the youngest group (54\%, 53\% and $44 \%$, respectively, descending in age) and a higher percentage of items were recalled at the beginning and end of the lists than were items in the middle of the lists. The interaction reflected the higher percentage of recall of fifth-position items in the experimental (48) than in the control (30) condition. A series of $t$ tests comparing performance between the two conditions at each serial position produced a significant difference only at the fifth position $[t(106)=4.52]$.

Figure 1 depicts the percent correct recall by the three groups separately at each serial position in each condition. An analysis was performed on the recall of fifth-position items by the three groups in each condition, to determine whether groups differed in their recall of those items. The main effects of groups and conditions were significant $[F(2,102)=5.68$ and $F(1,102)=21.6$, respectively $]$. The oldest group recalled more items than the two younger groups, and there was a higher recall of items when they were isolated than when they were not. A test of whether the isolation effect occurred for each group-that is, of whether significantly more items were recalled when isolated than when not isolated-revealed that the younger group demonstrated the effect $[t(34)=3.3]$, as did the intermediate age group [t(34) $=3.37]$, but that no significant isolation effect was obtained for the oldest group. The difference between the two conditions in the percent of fifth-position items recalled decreased with increasing age-namely, $25 \%, 18 \%$, and $11 \%$-thereby indicating a decrease in the effect. These results agree with those of 


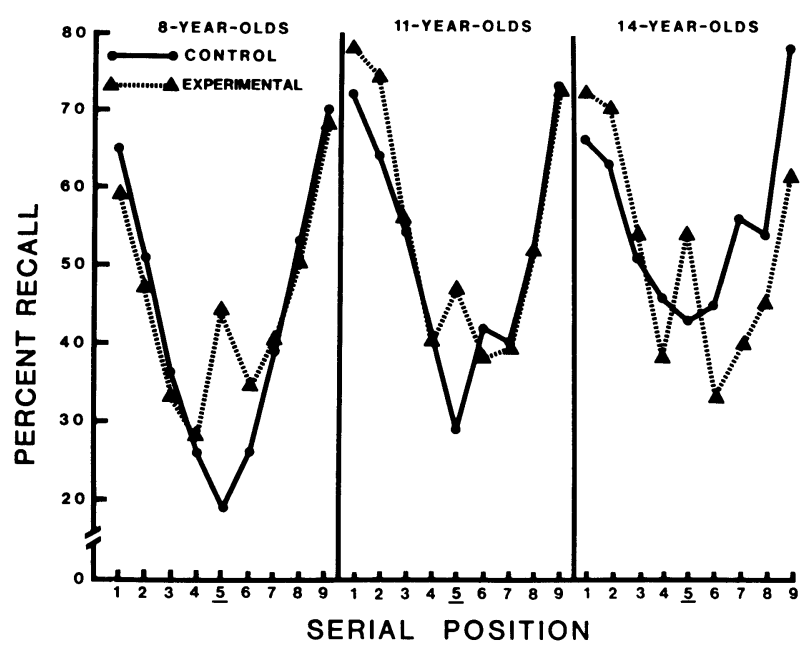

Figure 1. Percent of items correctly recalled by the 8-, 11-, and 14-year-olds at each serial position in each condition. The underlined position denotes the position at which the items were isolated.

Otto (1968) and Cimbalo et al. (1981), who also found a tendency for the effect to decrease as age increased.

To examine why the effect diminished as age increased, a closer evaluation was made of the number of fifth-position items that were correctly recalled by each subject, relative to that subject's total recall. This proportion reveals the efficiency with which items at the critical position were recalled by each group, independently of the performance by persons in other groups or conditions. The average proportion of items recalled in the fifth position by each group was $.11, .10$, and .11 for the experimental condition and $.05, .06$, and .09 for the control condition, ascending in age. Analyses of each condition revealed that, while the proportion of recall of fifth-position items did not differ among the groups in the experimental condition, it did differ in the control condition $[F(2,51)=4.41]$.

The 14-year-olds recalled a reliably higher proportion of fifth-position items in the control condition than did the 8-year-olds, with the 11-year-olds intermediate. Thus, the dissipation of the isolation effect was not a function of the relative recall of the items when they were isolated, but of the relative recall of those items when not isolated. The results for the 7-year-old first-graders are consistent with the above findings. Their proportion of recall of isolated items was .11, similar to that for the three older groups, but it was only .04 for control items, less than that for the older groups.

\section{Discussion}

Our finding that the isolation effect varies inversely with age is consistent with the findings of Otto (1968) and Cimbalo et al. (1981). Our results also indicate that one reason for this finding is that the proportion of fifth-position items recalled/total recall increased with age for the control condition but not for the experimental condition. This result leaves unanswered the question of whether the decrease with age of the isolation effect is a function of task difficulty (list length), or of differential responsiveness to the perceptual isolation. This issue can be examined further through making the level of task difficulty more appropriate for each age group. If, on the one hand, it is decreasing responsiveness to the perceptual factors that causes decreases in the size of the isolation effect as age increases, the size of the effect should vary independently of amount recalled. That is, a decrease in the recall of longer lists should not influence the size of the effect. If, on the other hand, task difficulty plays a primary role, then by equating for task difficulty, the difference between isolated and nonisolated items should remain relatively constant across age groups, or even increase with age. Experiment 2 was conducted to distinguish between these two competing hypotheses.

\section{EXPERIMENT 2}

\section{Method}

Subjects. There were 72 students in the study, 36 in each of two age groups from the same school district as in Experiment 1. The younger group had a $\overline{C A}$ of 11.80 years (.47); the older group had a $\overline{C A}$ of 13.97 years (.30). The respective $\overline{C A} \mathrm{~s}$ of these two groups and, respectively, of the two older groups in Experiment 1 were not significantly different. Students in the lower age group were from the 5th and 6th grades; those in the older age group were from the 7th and 8th grades.

Stimuli and Procedure. The stimuli were the same words that were used in Experiment 1. They were cast into two sets. In one set there were 11 words in 7 separate lists, for a total of 77 words; in the other set there were 13 words in 6 separate lists, for a total of 78 words. The 11-year-old group received the 11-word lists over seven trials, and the 13-year-old group received the 13-word lists over 6 trials. (The 8-yearolds in Experiment 1 had received the 9-word lists over 9 trials.) In all other respects, the procedure was identical to that in Experiment 1, with half of the students in each age group serving in either the experimental condition [isolated (red) item in middle list position] or control condition [all items same color (blue)]. Half the subjects in each condition were male (female). There were 7 orders of the 11 -word list and 6 orders of the 13-word list.

\section{Results}

Comparisons of amount recalled by the 11- and 13-yearolds on the 11- and 13-item lists in this study and the 8year-olds from Experiment 1 on the 9-item list revealed that groups differed reliably $[F(2,102)=13.64]$, with the 8-year-olds and the 11-year-olds recalling a higher proportion of items (.44 and .41 , respectively) than did the 13 year-olds (.36).

The isolation effect was demonstrated by each group $[t(34)=3.3,4.49$, and 5.26, ascending in age $]$; it is shown graphically in Figure 2 . A groups $\times$ conditions analysis of the percent of medial-position items that were correctly recalled revealed that the groups were similar $(31 \%, 34 \%$,

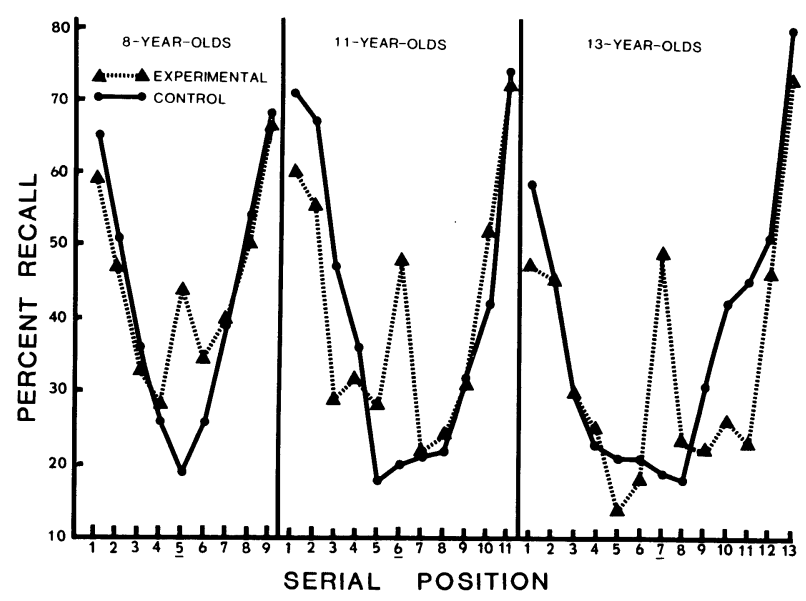

Figure 2. Percent of items correctly recalled by the 8-, 11-, and 13-year-olds at each serial position in each condition. The underlined position denotes the position at which the items were isolated. 
and $34 \%$, ascending in age) but that conditions differed $[F(1,102)=51.63]$, with $47 \%$ and $20 \%$ of the midposition items recalled in the experimental and control conditions, respectively. A series of $t$ tests at all of the other serial positions for each group produced significantly higher recall in the control than in the experimental conditions at the 3rd serial position for the 11-year-olds and at the 10 th and 11th serial positions for the 13-year-olds.

Difference in the percent recall between items when isolated and when not were reliable for each group, being $25 \%, 28 \%$, and $30 \%$ for the three groups, ascending in age. These modest age differences in the isolation effect were not statistically reliable.

To measure the influence of the isolation effect on recall, we calculated the proportion of midposition items recalled relative to total recall. In Experiment 1 , the proportion of isolated items recalled was fairly constant across age groups, but when items were not isolated, the proportion increased as age increased. In this experiment, the proportions were the same for the three groups when items were isolated (.11) and similar when items were not isolated $(.05, .04$, and .04 for the groups, ascending in age). Separate analyses for midposition items when isolated and when not isolated indicated that the groups did not differ in the proportion of items recalled whether isolated or not. These proportions resemble those of the 7year-olds in Experiment 1 (.11 and .04, respectively).

\section{Discussion}

Changing the list length and, consequently, the level of task difficulty produced similarities in the degrees of the isolation effect among all of the groups. These similarities resulted primarily because of changes in performance in the control condition. In the critical list position, the proportioni of recall of nonisolated items to total recall was directly related to overall recall performance, a function of list length, whereas the proportion of recall of isolated items was .10 or .11 , regardless of list length $(9,11$, or 13 items) or age (7-14 years). The consistent proportion of isolated-item recall across list length suggests that, within these list and age parameters, the cue to increased recall of the isolated items is perceptual and relatively invariant across these age groups. The similar- ity in the extent of the isolation effect-that is, the difference in recall between heterogeneous and homogeneous items when task difficulty was taken into account-suggests that both memorial and perceptual processes influence the size of the effect. To our knowledge this is the first evidence for the influence of memory capacity on this perceptual phenomenon.

Consequently, in order to make the task equivalent over age groups-a requirement if we are to isolate certain mechanisms of memorial processes influenced by memory capacity - task difficu'ty should vary directly with the age of the subject group. This would explain the differences between our results in Experiment 2 and those of Cimbalo et al. (1981) and Otto (1968).

\section{REFERENCES}

Calkins, M. W. (1894). Association. Psychological Review, 1, 476-483.

Carroll, J. B., Davies, P., \& Richman, B. (1971). The American Heritage word frequency book. Boston: Houghton Mifflin.

Cashen, V. M., \& Leicht, K. L. (1970). Role of the isolation effect in a formal educational setting. Journal of Educational Psychology, 61, 484-486.

Cimbalo, R. S., NowaK, B. I., \& Soderstrom, J. A. (1981). The isolation effect in children's short term memory. Journal of General Psychology, 105, 215-223.

Flavell, J. H. (1970). Developmental studies of mediated memory. In H. W. Reese \& L. P. Lipsitt (Eds.), Advances in child development and behavior (Vol. 5, pp. 182-211). New York: Academic Press.

Gabassi, P. G. (1980). Rehearsal and isolation effect in recall. Perceptual \& Motor Skills, 50, 603-606.

Hershberger, W. A., \& Terry, D. F. (1965). Typographical cuing in conventional and programmed text. Journal of Applied Psychology, 49, 55-60.

JONES, J. K. (1965). Colour as an aid to visual perception in early reading. British Journal of Educational Psychology, 35, 21-27.

Oтто, W. (1968). Elementary pupils' use of cues in paired-associate learning. Psychology in the Schools, 5, 178-182.

Pezdex, K. (1983). Memory for items and their spatial locations by young and elderly adults. Developmental Psychology, 19, 893-900.

Rabinowitz, F. M., \& ANDREWs, S. R. (1973). Intentional and incidental learning in children and the von Restorff effect. Journal of Experimental Psychology, 100, 315-318.

WALlaCE, W. P. (1965). Review of the historical, empirical, and theoretical status of the von Restorff phenomenon. Psychological Bulletin, 63, 410-424.

(Manuscript received November 19, 1988.) 